\title{
Pandoraea sputorum
}

National Cancer Institute

\section{Source}

National Cancer Institute. Pandoraea sputorum. NCI Thesaurus. Code C119327.

A species of gram-negative, non-fermenting rod-shaped bacteria belong ing to the genus

Pandoraea that has been isolated in human sputum. 